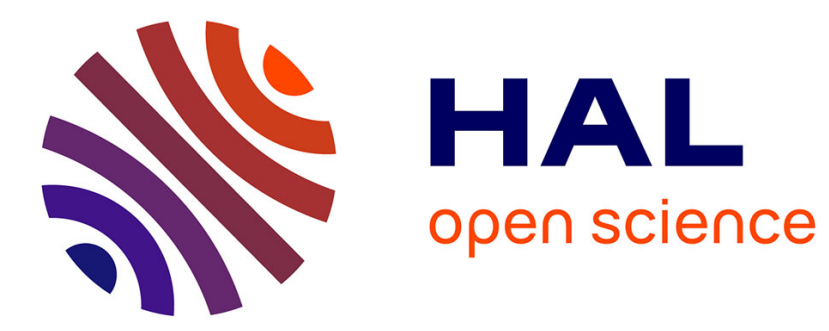

\title{
Modification of light sources for appropriate biological action
}

\author{
R Kozakov, H Schöpp, St Franke, C Stoll, D Kunz
}

\section{To cite this version:}

R Kozakov, H Schöpp, St Franke, C Stoll, D Kunz. Modification of light sources for appropriate biological action. Journal of Physics D: Applied Physics, 2010, 43 (23), pp.234007. 10.1088/0022$3727 / 43 / 23 / 234007$. hal-00597821

\section{HAL Id: hal-00597821 \\ https://hal.science/hal-00597821}

Submitted on 2 Jun 2011

HAL is a multi-disciplinary open access archive for the deposit and dissemination of scientific research documents, whether they are published or not. The documents may come from teaching and research institutions in France or abroad, or from public or private research centers.
L'archive ouverte pluridisciplinaire HAL, est destinée au dépôt et à la diffusion de documents scientifiques de niveau recherche, publiés ou non, émanant des établissements d'enseignement et de recherche français ou étrangers, des laboratoires publics ou privés. 


\title{
Modification of light sources for appropriate biological action
}

\author{
R Kozakov†, H Schöpp †, St Franke†, C Stoll $\ddagger$ and D Kunz $\ddagger$ \\ † Leibniz Institute of Plasma Science and Technology, Felix-Hausdorff-Str. 2, \\ D-17489 Greifswald, Germany \\ ‡ Charité - Universitymedicine Berlin, Sleep Research \& Clinical Chronobiology, Gr. \\ Hamburger Str. 5-11, D-10115 Berlin, Germany
}

\begin{abstract}
The impact of the non-visual action of light on the design of novel light sources is discussed. Therefore possible modifications of lamps dealing with the spectral tailoring and their action on melatonin suppression in usual life situations are investigated. Results of melatonin suppression by plasma lamps are presented. It was shown that even short-time exposure to usual light levels in working areas has an influence on the melatonin onset.

E-mail: kozakov@inp-greifswald.de

PACS numbers: 87.18.Yt, 87.50.W-
\end{abstract}

Submitted to: J. Phys. D: Appl. Phys. 


\section{Introduction}

The influence of light on the melatonin secretion in humans was discovered almost 30 years ago (Lewy et al. 1980). Approximately 20 years later the discovery of a novel nonvisual light receptor in human eyes followed (Berson et al. 2002). The new receptor, the so called intrinsically photosensitive retinal ganglion cell (ipRGC) was found to be responsible for the entrainment of human endogenous circadian rhythms with the natural 24 hours light variation. A novel photosensitive pigment melanopsin which is contained in ipRGC was discovered even earlier (Provencio et al. 2000). The action spectrum of melatonin suppression was measured experimentally in works of Brainard and Thapan (Brainard et al. 2001, Thapan et al. 2001). This spectrum is believed to be mainly influenced by the sensitivity spectrum of ipRGCs but other photo receptors seem also to have influence on the non-visual responce (Rea et al. 2005) These results have shown that the light plays a major role in the entrainment of suprachiasmatic nucleus which is considered as master clock of biological rhythms in humans. Many other studies have shown that the disturbance and especially the chronical disturbance (for example at shift workers) of biological rhythms can be a reason for several diseases like cardiovascular dysfunctions (Akerstedt et al. 1984), cancer (Levi 2000) and seasonal affective disorder (SAD)(Avissar et al. 1999). It is therefore important to consider the non-visual effects which arise when artificial light is delivered during the evening and night periods of the day. Furthermore several questions arise for future artificial illumination installations, like:

- Which spectra are best suited for evening and morning illumination respectively?

- What are the best intensity levels in the morning and in the evening?

- Which adverse reactions can occur or may lead to diseases?

- Which lamp technology is best suited for biologically efficient illumination - plasma based [fluorescent lamps (FL), high intensity discharges (HID)], incandescent or LED?

- What are the methods of investigation to determine the "best"?

Many published results explain more or less detailed investigations, but up to now no summarized knowledge is published.

The findings of Brainard and Thapan allowed Gall (Gall \& Lapuente 2002) to introduce a so called circadian metric of light which enables one to estimate the biological action of light in terms of melatonin suppression. The Gall model defines an action spectrum $s_{m s}$ (figure 1) based on the measurements of Brainard and Thapan. The $s_{m s}$ function is shifted relative to $V_{\lambda}$ to the short-wavelength region of the spectrum and has a maximum at approximately $460 \mathrm{~nm}$. The melatonin suppression action factor $a_{\mathrm{ms}} \mathrm{v}$ is then defined as

$$
a_{\mathrm{ms} \mathrm{v}}=\int_{380}^{580} s_{m s} S_{\lambda} \mathrm{d} \lambda \cdot\left(\int_{380}^{780} V_{\lambda} S_{\lambda} \mathrm{d} \lambda\right)^{-1},
$$

where $S_{\lambda}$ is the spectral irradiance and $V_{\lambda}$ is the spectral luminous efficiency. The values of $a_{\mathrm{ms} \mathrm{v}}$ range for Planckian radiators with colour temperatures (CCT) between 2000 
and $13000 \mathrm{~K}$ from 0.1 to 1.3 . The biologically effective illuminance level can be defined as

$$
E_{\mathrm{C}}=a_{\mathrm{ms} \mathrm{v}} \cdot E
$$

where $E$ is the usual photopic illuminance. Because the maximum of $s_{m s}$ lies in the shortwavelength region there exists a correlation between CCT and $a_{\mathrm{ms} \mathrm{v}}$ leading to higher $a_{\mathrm{ms}} \mathrm{v}$ values for higher CCT values. However this correlation is not unambiguous. Spectra with the same CCT can have different values of $a_{\mathrm{ms}} \mathrm{v}$ varying in distinct limits (Kozakov et al. 2008).

The non-visual metrics of Gall is a first attempt to quantify the biological action of light. Such a quantification is needed to predict the biological effectiveness of lighting and will help in future the lighting industry and architecture to design biologically "friendly" light sources. It should be noted that the Gall model of non-visual action of light is only an approximation based on the measurements of nocturnal melatonin suppression. Non-visual action of light has many aspects like for example sleep quality, alertness and others. In addition, the timing of light exposure is very important for nonvisual action. The photosensitivity of melatonin suppression is believed to be modulated over the day with lower sensitivity during subjective daytime. However unlike some

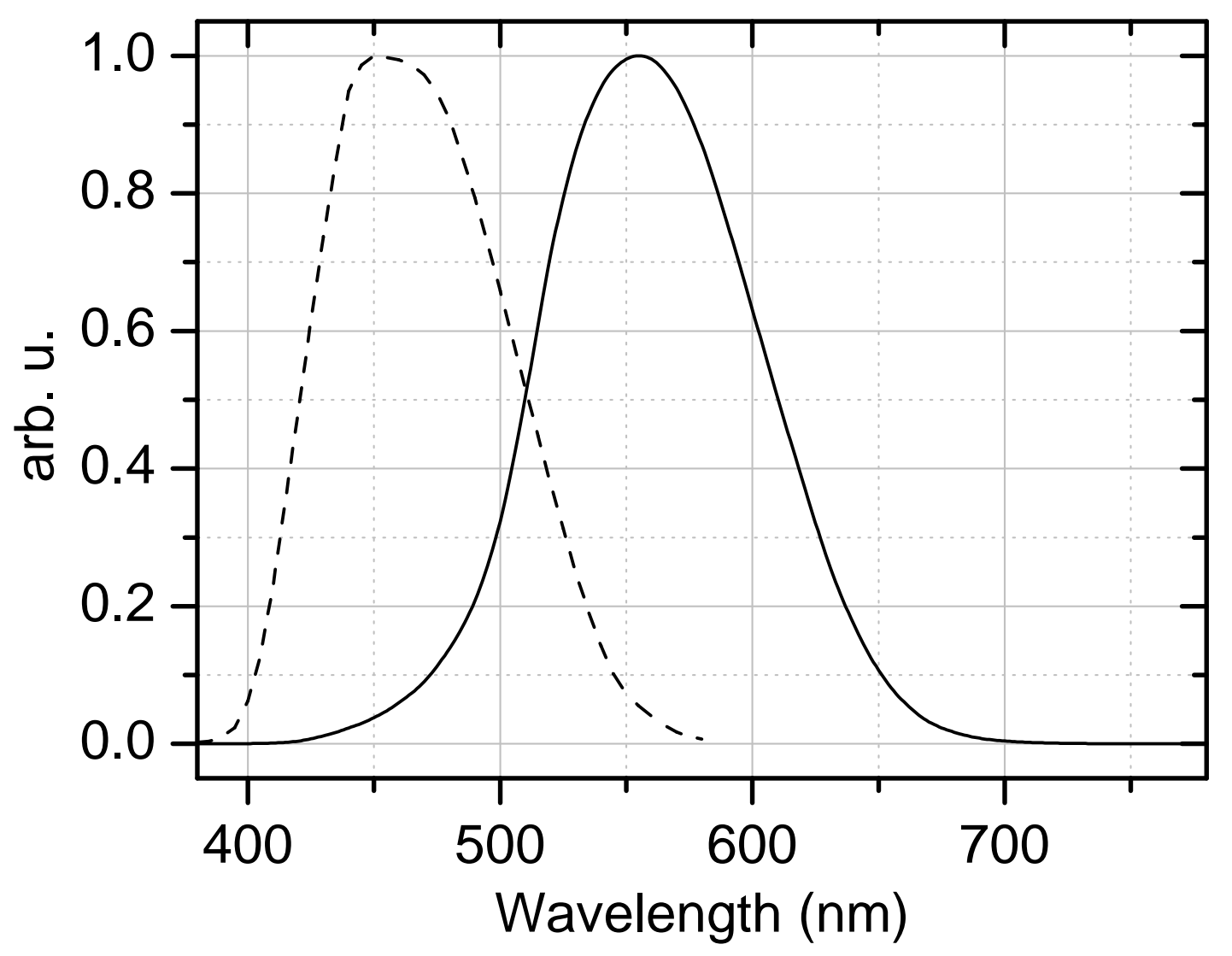

Figure 1. Comparison of spectral luminous efficiency $V_{\lambda}(-)$ and melatonin suppression action spectrum $s_{m s}$ (- - -) according to (Gall \& Lapuente 2002). 
rodent species no "dead zone" in photosensitivity during the day could be identified in humans (Jewett et al. 1997).

To be able to predict the exact effect of lighting on health one needs the adequate model based on the comprehensive understanding of non-visual light action. Such action of light seems to be much more complicated than the Gall model can describe. There exist indications that the polychromatic and monochromatic radiation can have different total effect on melatonin suppression (Figueiro et al. 2006a). Furthermore the duration of light exposure does not contribute linearly to the effective dose (stimulus) obtained by a person (Rimmer et al. 1995). Because melatonin suppression action spectrum (Brainard et al. 2001, Thapan et al. 2001) does not coincide with the spectral sensitivity of ipRGCs (Gamlin et al. 2007) it can be assumed that other receptors in retina contribute also to the non-visual action of light, see for example (Rea et al. 2005).

Nevertheless the current understanding of non-visual action of light is sufficient to define some standard evaluation situation for light sources under test. The German standardisation institute (DIN) holds regular meetings on this topic. Recently a preliminary standard on non-visual action of light sources was published (DIN V 5035100 2009) where the definition of terms considering the non-visual action is performed.

For a refinement of existing models and a development of new ones extensive experimental investigations of melatonin suppression are necessary. Most of experimental data on melatonin suppression were obtained in strict laboratory conditions. The applicability of models should be proven also for usual lighting situations. The paper represents an attempt to investigate systematically the influence of lighting conditions on melatonin suppression. For the investigations the commercially available luminaries were taken, equipped with lamps of commercially available wattages and placed in usual application position. Hence the light levels used in current work were not specially adjusted. The measured values of vertical illuminance at cornea lie between 80 and $500 \mathrm{~lx}$. The obtained values coincide with the recommended illuminance levels for work places according to (EN12464-1:2003-03 2003). For example bathrooms (200 lx), offices (300-750 lx) and lecture halls (500 lx). However one should mention that the recommended values are given for the illumination of working surfaces and the real illuminance at cornea can be as much as 5 times smaller (Figueiro et al. 2006b). Thus the illuminance values in the present work describe rather the situations of evening activities in well illuminated areas such as sporting halls, shopping malls, offices etc. than the residential environment where much lower illuminance values can be expected (Figueiro et al. 2006b). However some of the lighting conditions used in the present paper, for example bathroom like illumination with fluorescent lamps and illuminancies about 100 $\mathrm{lx}$, can be considered to be representative for bathroom illumination, especially after the ban of incandescent lamps in some parts of the world.

The results were obtained by tests of plasma light sources on the melatonin suppression during the evening melatonin onset. The commercially available light sources have been investigated and modified for proper support of natural biological rhythms. In the first stage of investigation the commercially available light sources 
were tested in their effectiveness to suppress melatonin. In the second stage the light sources were modified and again tested for melatonin suppression. Section 2 of this article describes the experimental conditions and the light sources used for illumination. Section 3 deals with modifications of light sources. In section 4 the results are discussed. Section 5 gives some hints for possible modification of light sources which were not investigated in present work. Finally a short summary is given.

\section{Experimental conditions}

\subsection{Measurement paradigm}

The cyclic rhythm of melatonin concentration is a robust marker of circadian rhythms. Therefore the investigation of the influence of artificial lighting in several lighting situations on melatonin concentration was performed. Three types of lamps were used for this purpose: the tubular fluorescent lamps (FL), the high intensity discharge lamps (HID) and a novel lamp based on the dielectric barrier discharge technology with Xe filling (Xe-DBD). All these lamps were subject for modifications in terms of their spectral power densities.

The melatonin measurements were performed in a sleep laboratory. In a randomized cross-over design the groups of healthy subjects (usually $\mathrm{N}=7$ to 10 per group, totally 28 persons, age 18-35 years) were exposed to 30 minutes of light starting one hour before habitual bedtime. Starting one week before the tests the subjects were asked to keep constant sleep times. Questionnaires (Pittsburgh Sleep Quality Index) allowed to determine the bed-times which were used to distribute the subjects into the 3 person groups with nearly identical bed-times. No subjects were of extreme chronotype.

Saliva samples were collected every 30 minutes before and after the light exposure and every 10 minutes during the light exposure, including one sample 10 minutes just before the lights-on and one sample 10 minutes just after the lights-off. Samples collection started 2 hours before lights-on and ended 30 minutes after lights-off. Melatonin concentrations were defined by radioimmunoassay with the least detectable dose of $\pm 0.15 \mathrm{pg} / \mathrm{ml}$.

The usual artificial lighting environments were chosen to investigate the influence of everyday lighting situations on the melatonin suppression. The arrangement of the luminaries was chosen to correspond to usual applications of light sources, like for example bathroom arrangement, office arrangement, hall lighting arrangement. The list of light sources used and their application in the corresponding lighting situations is given in table 1. During light exposure subjects were standing on assigned spots and facing the wall in front of them. Depending on specific luminaire they were looking either at the illuminated wall or at the luminaire itself. The luminance in their field of view was measured with a calibrated luminance measurement camera.

The spectrum of light was recorded with the help of a compact spectroradiometer (EPP-2000, StellarNet) in the range between 300 and $800 \mathrm{~nm}$. The illuminance 
Table 1. List of used light sources and lighting conditions.

\begin{tabular}{llll}
\hline $\begin{array}{l}\text { Lighting } \\
\text { condition }\end{array}$ & FL & HID & Xe-DBD \\
\hline Bathroom fixture & $\times$ & - & $\times$ \\
Office fixture (ceiling fixtures) & - & - & $\times$ \\
Hall fixture (ceiling floodlight) & - & $\times$ & - \\
Hall fixture (downlight) & - & $\times$ & - \\
\hline
\end{tabular}

Table 2. List of lamps used in initial tests. RaCIE is colour rendering index. E is vertical illuminance corneal, $a_{\mathrm{ms}} \mathrm{v}$ is the biological action factor, equation (1).

\begin{tabular}{lllllll}
\hline Lamp & Luminaire & E [lx] & Ec & CCT $[\mathrm{K}]$ & RaCIE & $a_{\mathrm{ms} \mathrm{v}}$ \\
\hline FL6000 & Bathroom & 130 & 91 & 5500 & 80 & 0.70 \\
HID 70W & Hall downlight & 500 & 245 & 4200 & 65 & 0.49 \\
Xe-DBD & Bathroom & 500 & 200 & 2900 & 67 & 0.40 \\
\hline
\end{tabular}

was measured with the help of a illuminance meter (PocketLux 2, LMT). These measurements were performed at the positions of the eyes of subjects (at cornea). For each tested lighting situation tested a dim-light situation $(\leq 1 \mathrm{~lx})$ was also randomly included in test. During a dim-light situation the subjects underwent the usual routine with dim-light.

The melatonin suppression percentage was defined as the difference between melatonin variations in dim light situation and tested light situation related to the absolute value of melatonin in dim light condition 1 hour before habitual bedtime (instant of light-on):

$$
S=\frac{\Delta M_{D I M}-\Delta M_{T E S T}}{M(B T-1)},
$$

where $M(t)$ - melatonin concentration as function of time, $\Delta M=M$ (lights-off) $M$ (lights-on), $B T$ - habitual bedtime in hours. The choice of this suppression definition is defined by the work of (Thapan et al. 2001) where a similar formulation was used for the determination of the melatonin suppression level. The experimental design in that work was similar to that in our project - 30 minutes of light delivered during dim light melatonin onset. The dim and light situations were compared by paired two-tailed $\mathrm{t}$ test applied to the melatonin change rate calculated as $\Delta M / M(B T-1)$.

\subsection{Light sources at stage 1}

In the first stage of investigation the commercially available lamps were tested. The list of the lamps and their characteristics are given in table 2. The spectra of these lamps are shown in figure 2 . 


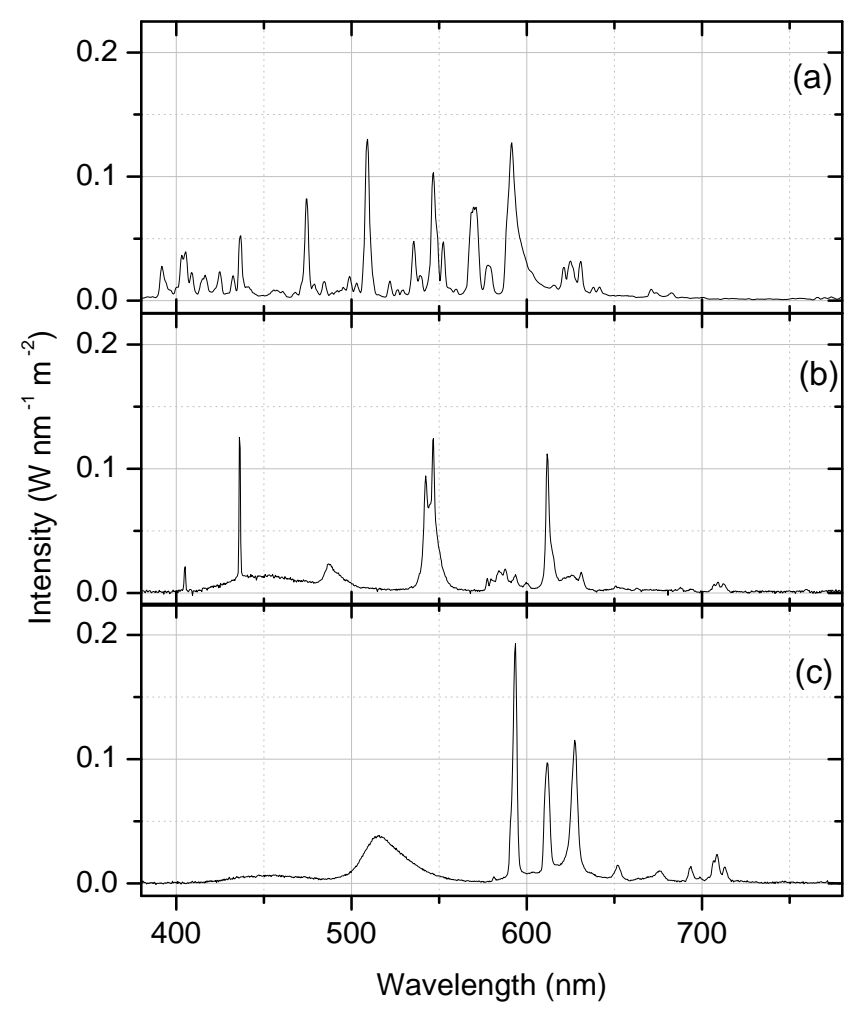

Figure 2. Spectra of lamps used in stage 1 tests (see table 2). (a) - HID 70W, (b) FL6000, (c) - Xe-DBD.

Table 3. List of tested modified lamps. RaCIE and RaDIN are colour rendering indexes calculated according to CIE and DIN standards respectively (see section 4). E is vertical illuminance.

\begin{tabular}{lllllllll}
\hline Lamp & Type & Modification & E $[\mathrm{lx}]$ & $\mathrm{Ec}$ & $\mathrm{CCT}[\mathrm{K}]$ & RaCIE & RaDIN & $a_{1}$ \\
\hline FL1800 & evening & combination of phosphors & 80 & 8.8 & 1800 & 77 & 81 & 0. \\
FL10000 & morning & combination of phosphors & 100 & 108 & 10000 & 80 & 80 & 1. \\
HID250 W & morning & additional indium & 450 & 369 & 5500 & 81 & 82 & 0. \\
Xe-DBD 1500 & evening & combination of phosphors & 100 & 10 & 1500 & 63 & 74 & 0. \\
\hline
\end{tabular}

The spectrum of HID 70W (NARVA NCT 70W nw) lamp is typical for HID lamps and consists of radiation of metals included in lamp filling. The FL6000 (NARVA 18WT8 860) lamp and Xe-DBD (OSRAM) lamp show typical fluorescent spectra consisting of radiation of phosphors and in the case of FL6000 mercury lines. At the beginning of the stage one there was no Xe-DBD lamp for general illumination available. The lamp for technical illumination purposes was used. Therefore the colour rendering of this lamp as read from table 2 is not optimized for the purposes of general illumination. It should however be noted that this lamp is fluorescent one and by proper combination and choice of phosphors very good colour rendering (up to $\mathrm{Ra} \geq 90$ ) can also be obtained. 


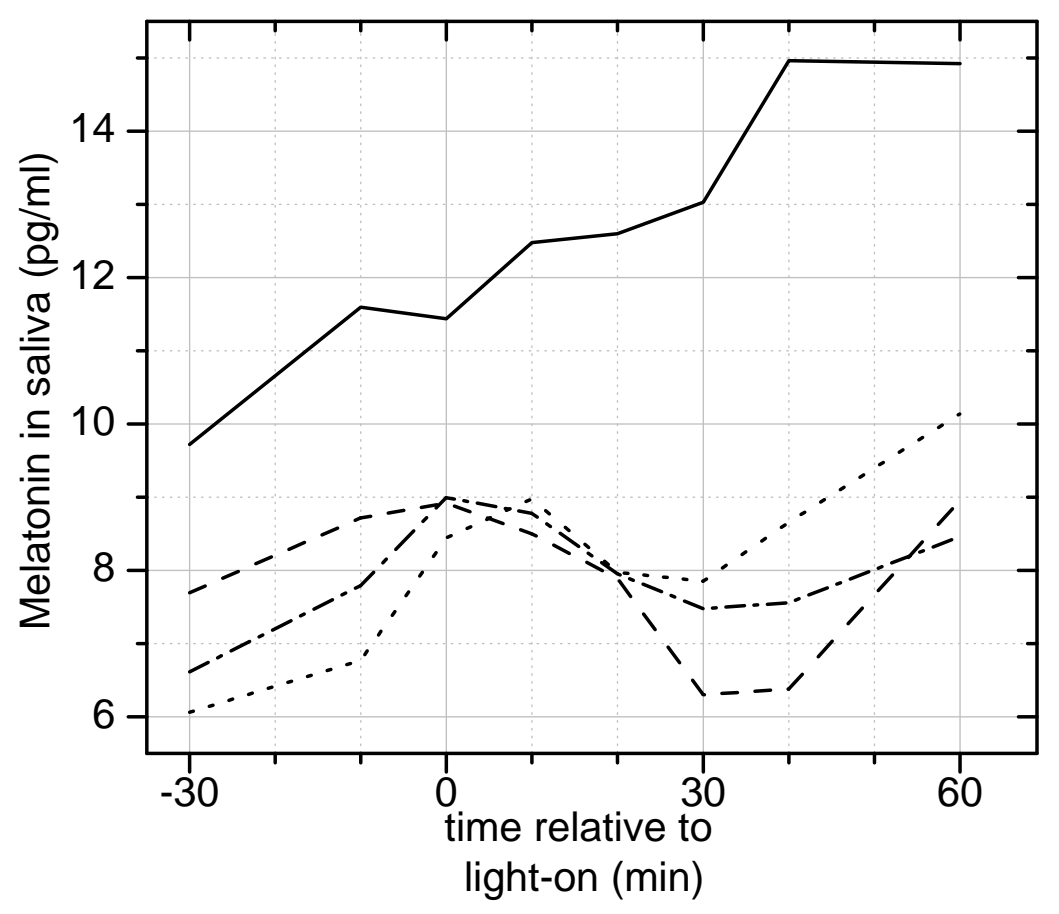

Figure 3. Example of the melatonin course during the evening (averaged over $\mathrm{N}=9$ subjects) (a) dim-light (—), HID 70W (- - -), FL6000 ( . . . . ) and Xe-DBD $(-\cdot-)$.

\subsection{Light sources at stage 2}

For the second stage of investigations modified lamps were manufactured. The list of the lamps and their characteristics are given in table 3. The fluorescent lamps with the $\mathrm{CCT}=1800 \mathrm{~K}(\mathrm{FL} 1800)$ and $\mathrm{CCT}=10000 \mathrm{~K}$ (FL10000), the Xe-DBD lamp with $\mathrm{CCT}=1500 \mathrm{~K}$ (Xe-DBD 1500), the $250 \mathrm{~W}$ HID lamp with additional amount of Indium (HID $250 \mathrm{~W})$.

\section{Light sources modifications}

The results of the initial investigations have shown that the investigated lighting conditions are active in the biological sense. An example of group-averaged melatonin course is shown in figure 3. The data in figure 3 show some scattering; for example the dim-light situation seems to be shifted relative to other light situations. Furthermore the curves do not coincide before the time "0". The differences may be ascribed to the intra-individual variations of melatonin course and photic history of subjects.

There are situations where biologically active illumination is undesirable, for example in the evening hours, when the natural onset of melatonin production takes place. During this period of time a short exposure to light, for example in the bathroom during preparation to sleep can cause a significant suppression of melatonin concentration in blood and thus reduce the readiness to sleep. On the other hand there 
exist situations where one would like to suppress the melatonin in order to raise the alertness and reduce the sleepiness, for example in the morning hours after awakening or for the light treatment of jet-lag. The best solution would be a single lamp with changeable spectrum. The use of "morning" and "evening" lamps would be also a possibility for proper biological lighting. Our project uses the latter option as its objective. In the following possible modifications of lamps under investigations are discussed.

\subsection{Fluorescent lamps}

Both usual tubular FL and the Xe-DBD type lamps belong to the fluorescent kind of lamps. Their spectra are mainly determined by the mixture of phosphors used in the lamp. Therefore it is possible to produce lamps with lower and higher values of $a_{\mathrm{ms}} \mathrm{v}$. The other constraints on spectrum modification are the good colour rendering index $(\mathrm{Ra}>80)$ and a small distance to the Planckian locus in the colour space (condition on white light).

The possibility to create a "morning" lamp can be accomplished easier than that of creating an "evening" lamp. As far as the maximum of $s_{m s}$ lies in the short-wavelength region of the visible spectrum the increase of blue phosphor fraction in the mixture of phosphors gives the desirable effect on $a_{\mathrm{ms} \mathrm{v}}$. Although attention should be paid to balance the short-wavelength radiation with others to keep good $\mathrm{Ra}$ and stay in the vicinity of Planckian locus. Such a modification can be performed in the frame of usual three-phosphor technology. Fig. 4(a) shows an example of the spectrum with the increased short-wavelength fraction of the radiation developed for the tests at stage 2 . The CCT of this lamp is equal to $10000 \mathrm{~K}$. Recently lamps with similar CCTs became commercially available.

The "evening" lamp represents a bigger challenge to overcome. To reduce the biological effectiveness of a lamp one has to reduce the radiation fraction in the shortwavelength region of the visible spectrum. On the one hand the elimination of shortwavelength radiation causes a difficulty in creating a white light which is only suitable for general lighting. The way to solve this problem lies in the chromatic adaptation possibility of human eye. Any radiator whose spectrum lies near enough to Planckian locus is perceived as "white". To reduce the biological effectiveness of light one needs to use the lower values of CCT. With the decrease of CCT the short-wavelength fraction of radiation of Planckian radiators and hence their biological activity diminishes. Fig. 4(b) shows the spectrum of such an "evening" lamp developed for the tests at stage 2.

On the other hand the almost complete elimination of short-wavelength light is a difficult task itself for the fluorescent lamps because of mercury spectral lines. By simple combination variation of three phosphors it is impossible to filter out those lines. Usual phosphors are more or less transmittent in that region and in order to perform such filtering a special blue-absorbing phosphor was developed and used in the second stage of investigations (see table 3). The abandonment of mercury is a 


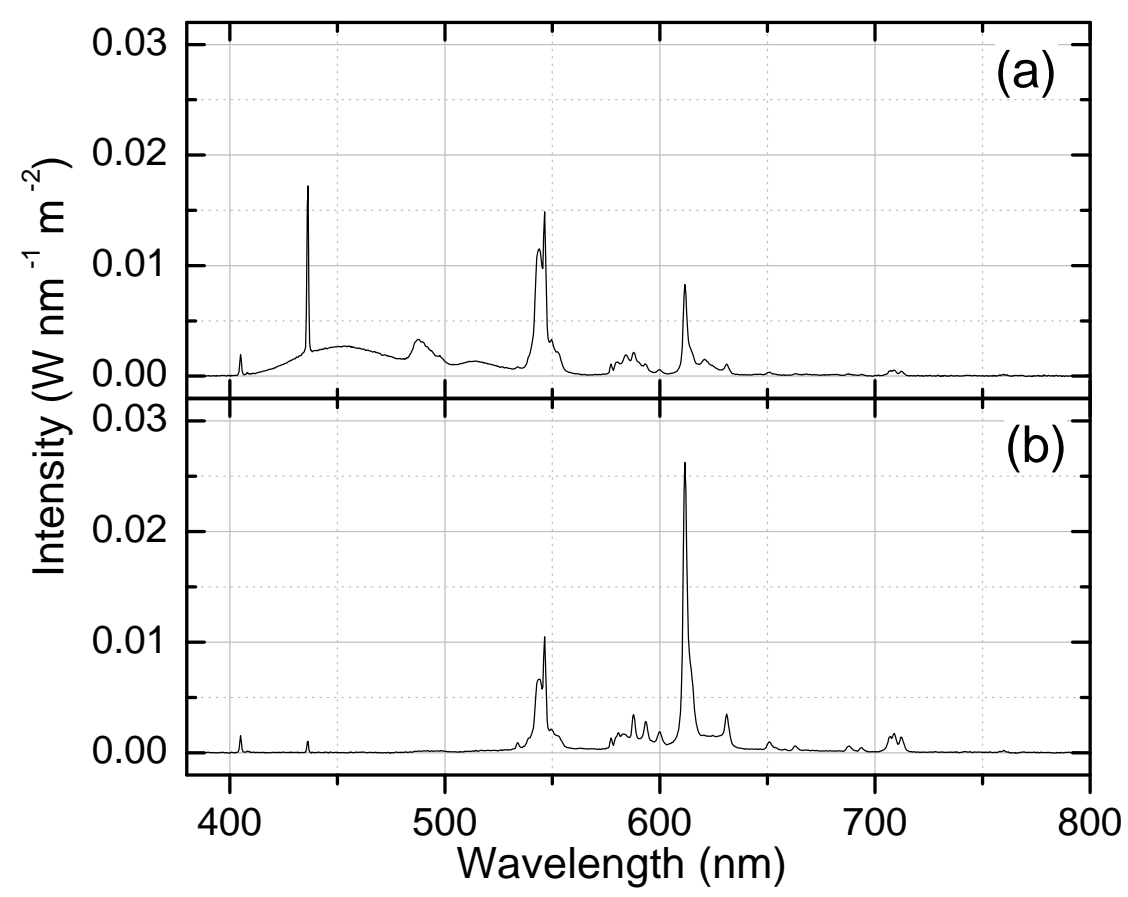

Figure 4. Example of spectra of the modified FL - "morning lamp" with $\mathrm{CCT}=10000 \mathrm{~K}(\mathrm{a})$ and "evening lamp" with CCT $=1800 \mathrm{~K}$ (b). In both cases the $\mathrm{Ra} \geq 80$.

challenging task for fluorescent lamps because of its very efficient conversion of electrical energy into resonant UV-radiation which is then converted into visible radiation. The presence of short-wavelength radiation is inherently persistent for all mercury based fluorescent lamps. One approach to overcome this problem is the Xe-DBD lamp. It also efficiently converts electric energy to the UV excimer radiation around $172 \mathrm{~nm}$. In typical Xe-DBD discharges the amount of visible radiation is negligible relative to the UV radiation. Therefore they are neutral in the sense of non-visual action and there exists a bigger freedom in choosing the combinations of phosphors because one does not have to account for the Xe contribution in colour mixing procedure. More details on science and technology of gas discharge lamps can be found for example in (van den Hoek et al. 2001, Lapatovich 2009).

\subsection{HID lamps}

The modification of spectra of HID lamps represents a difficult task because the light of HID lamps comes from the high pressure discharge arc directly. The filling composition takes effect on the physical conditions within the lamp like temperature and pressure and therefore on the properties of the visible radiation. Adding the salts of some elements does not necessarily lead to the appearance of their characteristic spectral lines in the spectrum of the lamp. Due to the changes in the physical and chemical composition of the vapours the spectra may change completely, for example by adding a readily 


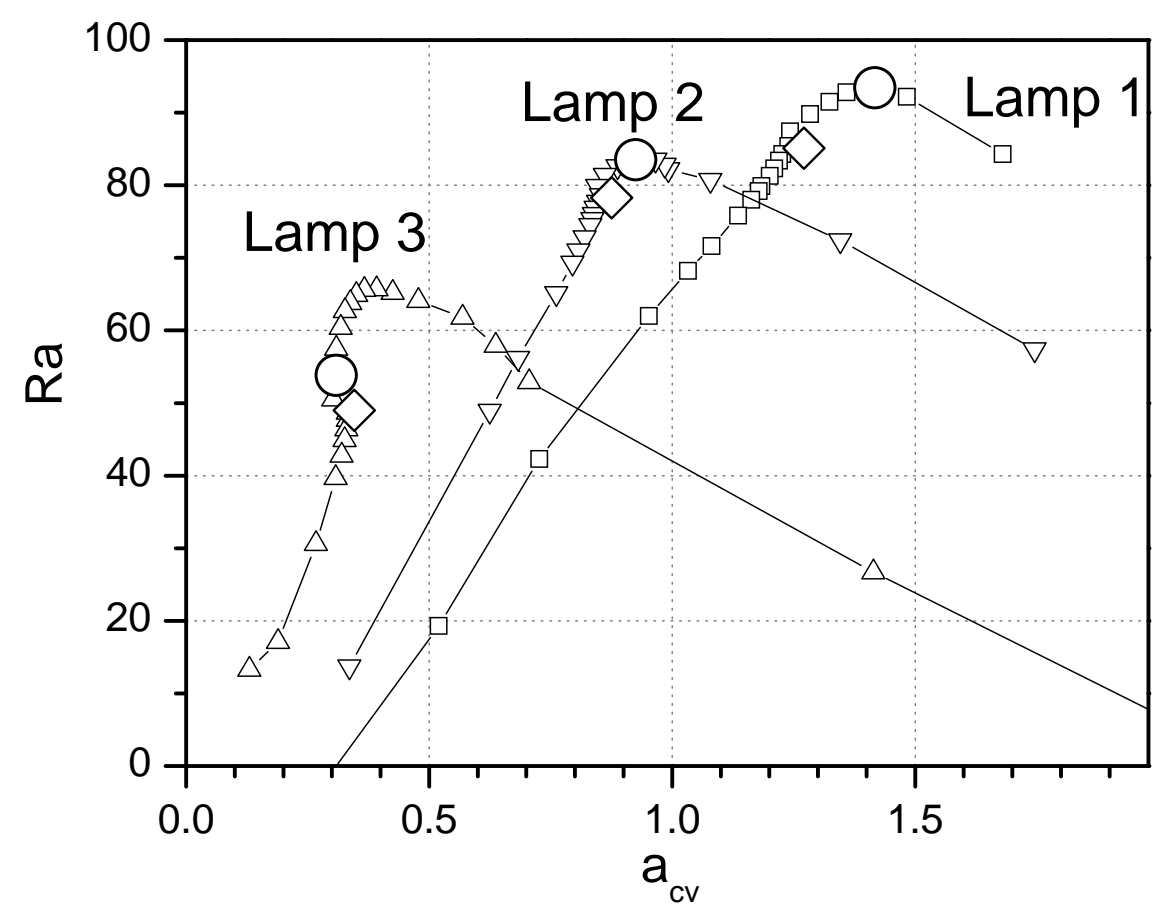

Figure 5. Simulation of spectra modification by one Gaussian peak. The positions of the unmodified lamps are represented by open diamonds. The possible aims of modification are shown by open circles. The corresponding parameters are given in table 4 .

ionizable element suppresses the radiation of elements with higher lying energy levels.

Nevertheless it is possible to perform numerical simulations of the possible modifications of the spectra of HID lamps. By such a simulation the possible positions and intensities of additional spectral lines can be determined which should lead to the desirable changes in biological effectiveness of the lamp radiation. The similar strategy of "morning" and "evening" lamp can also be followed here. Again the objective of "morning" lamp can be reached easier than that of "evening" lamp.

On the one hand the element Indium has a very "convenient" resonant radiation at $451 \mathrm{~nm}$ which lies near the maximum of the action spectrum $s_{m s}$. Therefore by increasing indium amount in the lamp filling one can expect the increase of the biological activity of the lamp in sense of melatonin suppression.

On the other hand there are more options in reducing the biological effectiveness of an "evening" lamp. Not only the reduction of the radiation in the short-wavelength region, but also an increase of radiation in other spectral regions can lead to the desirable reduction of $a_{\mathrm{ms} \mathrm{v}}$.

In order to investigate the possible spectral ranges suitable for diminishing the $a_{\mathrm{ms}} \mathrm{v}$ value, the spectrum of a commercial HID lamp was taken and one additional Gaussian peak was numerically added. The position, amplitude and the width of the peak were variable. The values of those three parameters were scanned within reasonable limits yielding an overview of the possible modifications of the given spectrum by one 
Table 4. Results of modification of the spectrum of the HID lamp with one additional Guassian peak.

\begin{tabular}{lllllll}
\hline Lamp & Peak wavelength & FWHM & $\begin{array}{l}\text { Amplitude } \\
(\% \text { to max })\end{array}$ & CCT (K) & RaCIE & $a_{\mathrm{ms} \mathrm{v}}$ \\
\hline Lamp 1 & no peak & - & - & 10600 & 85.1 & 1.27 \\
Lamp 2 & no peak & - & - & 6041 & 78.3 & 0.88 \\
Lamp 3 & no peak & - & - & 2549 & 49 & 0.35 \\
Lamp 1 & $490 \mathrm{~nm}$ & $1 \mathrm{~nm}$ & 119 & 12080 & 93.4 & 1.39 \\
Lamp 2 & $505 \mathrm{~nm}$ & $1 \mathrm{~nm}$ & 210 & 6710 & 83.5 & 0.92 \\
Lamp 3 & $550 \mathrm{~nm}$ & $3 \mathrm{~nm}$ & 80 & 2910 & 53.7 & 0.31 \\
\hline
\end{tabular}

Gaussian peak. The resulting spectra whose chromaticity coordinates were lying near the Planckian locus were kept. As the result a figure of merit between colour rendering and biological effectiveness was obtained.

The spectra of three commercially available HID lamps: Nachroma (Lamp 1), VEG (Lamp 2) and Gold (Lamp 3) from NARVA G.L.E. were tested. The colorimetric and biologic properties of the lamps are summarized in table 4. The results of simulations in figure 5 show that depending on the initial filling of the lamp different combinations of $a_{\mathrm{ms} \mathrm{v}}$ and Ra are possible. For the particular lamps the significant increase or decrease in the biological effectiveness can be achieved only by a decrease of the colour rendering. The lamps 1 and 2 show a potential of being "morning" lamps and there exists a possibility of increasing both $a_{\mathrm{ms} v}$ and Ra. The lamp 3 which was considered as a candidate for "evening" lamp has a limited ability of decreasing $a_{\mathrm{ms}} \mathrm{v}$ while still keeping Ra higher than the initial value. The possible aim of modification is shown in figure 5 as open circles with corresponding parameters listed in table 4 . For lamps 1 and 2 the maximum of $\mathrm{Ra}\left(a_{\mathrm{ms}}\right)$ dependence is chosen as modification aim. Another possibility is the crossing point the curve with line $\mathrm{Ra}=80$ at the right wing of the curve. There even higher values of $a_{\mathrm{ms} \mathrm{v}}$ are possible at still high enough Ra. For lamp 3 the point was chosen where the $a_{\mathrm{ms}} \mathrm{v}$ value is approx $10 \%$ smaller and Ra value is approx 10\% higher than in the original spectrum as the possible aim of modification. Other options for reducing biological effectiveness of this lamp can only be achieved by significant decrease of colour rendereing.

\section{Melatonin suppression by modified lamps}

For the second stage of investigation the modified lamps were manufactured and tested for melatonin suppression. Lamps were installed in the usual lighting fixtures as shown in table 1. The list of lamps and modifications together with properties is given in table 3 . The lamps of fluorescent type were modified by changing the relative composition of three phosphors - blue, green-yellow and red. The lamps with low CCT additionally contained a phosphor which absorbs the radiation in the short-wavelength visible region. It was possible to obtain an $a_{\mathrm{ms}} \mathrm{v}$ as high as 1.08 and as low as 0.1 . The light sources 
with CCTs smaller than $2000 \mathrm{~K}$ are not perceived as "completely" white anymore. Although the Ra can be around 80 those lamp emit yellowish light. Due to chromatic adaptation their light can still be perceived as almost white and comparable to the candle light. With decreasing CCT the task of keeping high value of Ra becomes more and more difficult. This can be seen from the table 3 - the lamp with CCT $=1800$ can still have $\mathrm{Ra}=77$ but lamp with $\mathrm{CCT}=1500$ has only $\mathrm{Ra}=63$. It should however be noted that the evaluation of colour rendering properties of the lamps with such a small CCT is not a usual task. In some standards like for example German DIN there exists the lowest limit of $\mathrm{CCT}=2300 \mathrm{~K}$ for the reference light source which is used for calculation of colour rendering index. This fact yields the controversy between DIN (DIN 5033-31992) and CIE (CIE 13.3-1995 1995) colour rendering evaluation routines. For example the spectrum of Xe-DBD 1500 lamp has a Ra value 63 when calculated with CIE standard and Ra value of 74 when calculated with DIN standard with $2300 \mathrm{~K}$ reference illuminant. The values of Ra calculated with both standards are given in table 3 . One can see a discrepancy between both evaluation procedures for the lamps with lower correlated colour temperatures.

For the "morning" lamp there exist no difficulty with keeping Ra over 80. The HID lamp used here was the metal halide lamp with additional amount of indium. This allowed to achieve the $a_{\mathrm{ms}} \mathrm{v}$ value of 0.82 .

The results of melatonin suppression are shown in figure 6 according to equation (3). One can see that the measured melatonin suppression correlates well with the biologically effective illuminance level as defined by equation (2). The observed linear dependence can be considered to be a linear part of a logistic one which was used in works of Brainard and Thapan. But it should be noted that $E c$ values are only a rough estimate of the non-visual stimulus. In general it can be stated that the behaviour of melatonin suppression corresponds to the Gall model predictions - higher Ec values yield higher values of melatonin suppression.

\section{Other light sources}

The light sources other than plasma type ones were not covered in the scope of our investigations. In the frame of the melatonin suppression model of Gall it is nevertheless possible to give some recommendations about possible modifications of spectra of LEDs and incandescent lamps.

\section{1. $L E D$}

The RGB type LEDs can be considered as one of the most flexible light sources with changeable biological properties. The variation of the relative intensities of individual blue, green and red LEDs can generally give any value of $a_{\mathrm{ms}} \mathrm{v}$. The only constraints are the colour rendering properties and the condition for white light. The topic of optimization of LEDs radiation was already considered earlier, see e.g. (Žukauskas et al. 


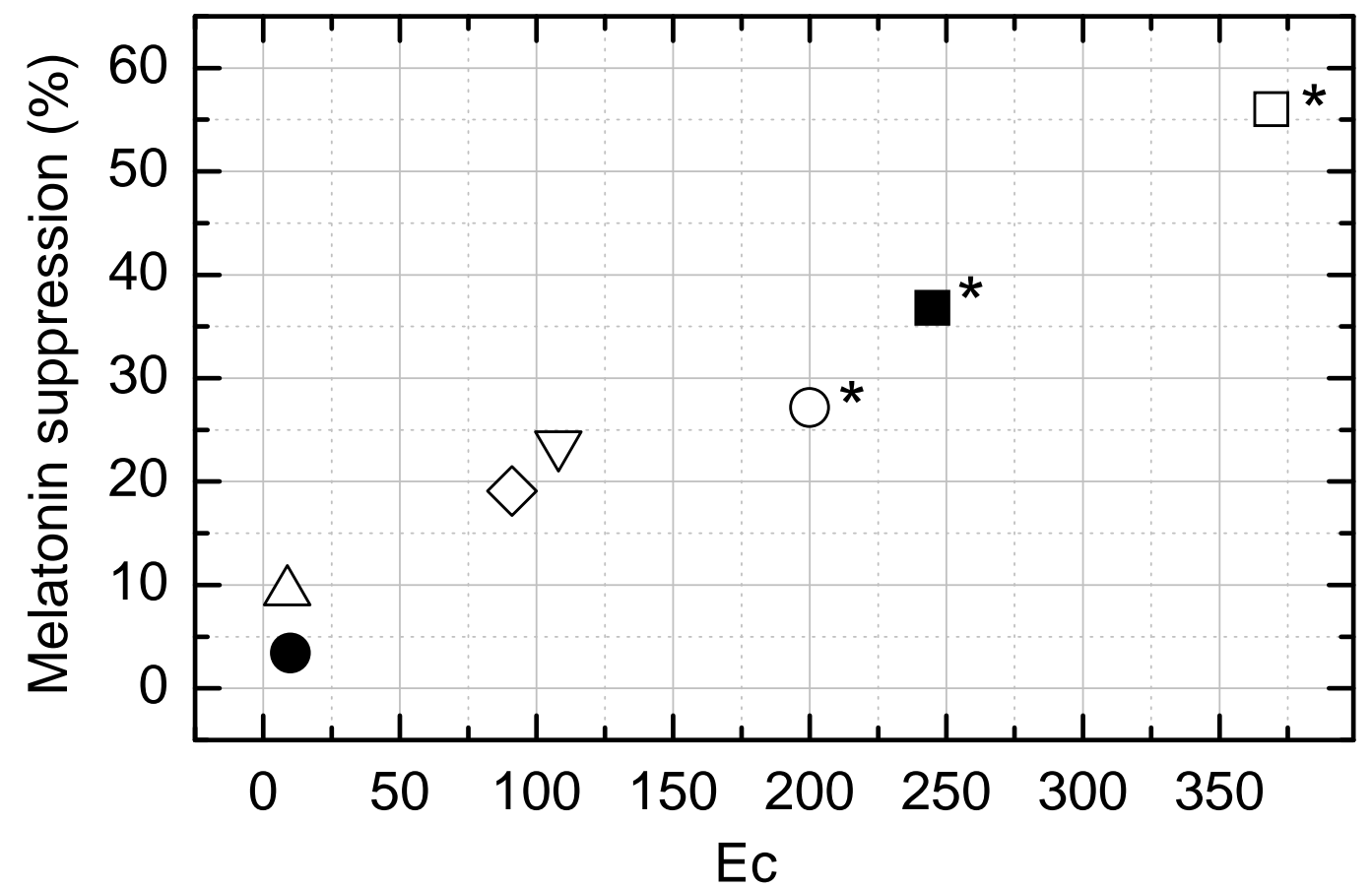

Figure 6. Comparison of melatonin suppression with initial and modified light sources. FL6000 $(\diamond)$, FL1800 $(\triangle)$ and FL10000 $(\nabla)$ - tubular fluorescent lamps in bathroom fixture; HID $70 \mathrm{~W}(\mathbf{\square})$ and $250 \mathrm{~W}(\square)$ - lamp with an additional amount of indium in the filling, ceiling fixture and ceiling floodlight respectively; Xe-DBD (O ) - lamp in bathroom fixture; Xe-DBD $1500(\bullet)$ - lamp in ceiling fixture, office arrangement. Asterisks indicate the statistically significant $(\alpha=0.05)$ difference in the melatonin change rate with respect to dim-light situation.

2002). The result of such optimization is a figure of merit between visual efficiency $\left(V_{\mathrm{S}}\right)$ and colour rendering (Ra). This curve shows that for given Ra (for example 80) a maximum achievable $V_{\mathrm{S}}$ exists. The knowledge about biological activity of light can be considered as a new constraint on the LED light source. The optimization procedure of spectral composition of LED radiation can be made with the additional condition that the biological action of the light should be equal to that of Planckian radiator with the same colour temperature. In (Kozakov et al. 2008) RGB-spectra have been optimized for maximum visual efficiency $V_{\mathrm{S}}$ with respect to the Ra. Set of different CCTs has been investigated. It was shown that additional constraint on the biological effectiveness of light reduces the maximum achievable $V_{\mathrm{S}}$.

\subsection{Incandescent lamps}

Spectra of incandescent lamps are very close to Planckian radiators with CCT values lying between 2700 and $3000 \mathrm{~K}$. Their spectrum contains a certain amount of radiation in the short-wavelength spectral range. The biological action factor calculated by equation (1) for an incandescent lamp has the value of 0.36 for $\mathrm{CCT}=2700 \mathrm{~K}$. A fluorescent lamp of type 827 with $\mathrm{CCT}=2700$ and $\mathrm{Ra}=80$ has an $a_{\mathrm{ms}} \mathrm{v}$ value of 0.25 . 
Therefore incandescent lamps can have higher biological action than fluorescent lamps at the same CCT and also suppress melatonin. Possible modification of incandescent lamps can be performed either with spectrally selective filters ("morning" lamp) or by reducing the lamp power and thus the temperature of the filament ("evening" lamp).

\section{Summary}

An overview of current status of research in the field of the non-visual action of light from the point of view of physics and lighting application was given. The ways of modification of light sources for a proper non-visual action were discussed. The results of the investigation on the influence of plasma based light sources on the suppression of evening melatonin production were presented. The concept of "evening" and "morning" lamps was introduced. Modified "evening" lamps have shown significantly smaller melatonin suppression than commercially available lamps. Some increase in the melatonin suppression have been obtained by the modified "morning" lamps. It was shown that short-time (30 minutes) exposure to lighting conditions as described in the introduction do effectively suppress melatonin. This result allows to define a standard procedure to quantify the biological effectiveness of light sources for future use in lighting applications.

\section{Acknowledgments}

This work was supported by German ministry of education and research under the grant BMBF FKZ13N8968-74. The authors wish to thank the companies Trilux, OSRAM, NARVA BEL, NARVA G.L.E., Litec LLL for the fruitful cooperation.

\section{References}

Akerstedt T, Knuttson A, Alfredsson L \& Theorell T 1984 Scandinavian journal of work environment and health 10, 409-414.

Avissar S, Schreiber G, Nechamkin J, Neuhaus I, Lam G K \& Schwartz P 1999 Archives of general Psychiatry 56, 178-183.

Berson D, Dunn F \& Takao M 2002 Science 295(5557), 1070-1073.

Brainard G, Hanifin J, Greeson J, Byrne B, Glickman G, Gerner E \& Rollag M 2001 Journal of Neuroscience 21(16), 6405-6412.

CIE 13.3-1995 1995 International commission on illumination.

DIN 5033-3 1992 Beuth Verlag Berlin, Germany.

DIN V 5035-100 2009 Beuth Verlag Berlin, Germany.

EN12464-1:2003-03 E N 2003 European norm EN12464.

Figueiro M G, Rea M S \& Bullough J D 2006 a Neuroscience Letters 406, 293-297.

Figueiro M G, Rea M S \& Bullough J D 2006b Journal of carcinogenesis 5(20), .

Gall D \& Lapuente V 2002 Licht (7-8), 860-871.

Gamlin P D R, McDouga D H, Pokorny J, Smith V C, Yau K W \& Dacey D M 2007 Vision Research 47, 946-954. 
Jewett M E, Rimmer D W, Duffy J F, Klerman E B, Kronauer R E \& Czeisler C A 1997 American Journal of Physiology - Regulatory Integrative and Comparative Physiology 273(5), R1800R1809.

Kozakov R, Franke S \& Schöpp H 2008 LEUKOS 4(4), 255-263.

Lapatovich W P 2009 Phys. Scripta T134, 014024.

Levi F 2000 Novartis foundation symposium (227), 119-136.

Lewy A J, Wehr T A, Goodwin F K, Newsome D A \& Markey S P 1980 Science 210(4475), 1267-1269.

Provencio I, Rodriguez I, Jiang G, Hayes W, Moreira E \& Rollag M 2000 Journal of Neuroscience 20(2), 600-605.

Rea M S, Figueiro M G, Bullough J D \& Bierman A 2005 Brain Research Reviews 50(2), 213-228.

Rimmer D W, Boivin D B, Shanahan T L, Vu B, Kronauer R E \& Czeisler C A 1995 Sleep Research $\mathbf{2 4 A}, 538$.

Thapan K, Arendt J \& Skene D J 2001 Journal of Physiology-London 535(1), 261-267.

van den Hoek W J, Jack A \& Luijks G M J F, eds 2001 Lamps Ullmann's Encyclopedia of Industrial Chemistry 6 edn Wiley-VCA Weinheim, New York.

Žukauskas A, Vaicekauskas R, Ivanauskas F, Gaska R \& Shur M S 2002 Applied Physics Letters 80, 234236. 